\title{
Alteration of lipid profile and prognostic value of lipids on the length of hospital stay in COVID-19 pneumonia patients
}

\section{Qin Chen}

Xiehe Affiliated Hospital of Fujian Medical University

\section{Minghan Huang}

Fujian University of Traditional Chinese Medicine

\section{Ziwen Zhao}

Xiehe Affiliated Hospital of Fujian Medical University

Yukun Luo ( $D$ luoyukun@hotmail.com )

https://orcid.org/0000-0002-9741-1687

\section{Research}

Keywords: COVID-19 pneumonia, lipids profile, cholesterol, length of hospital stay, infection

Posted Date: July 1 st, 2020

DOI: https://doi.org/10.21203/rs.3.rs-30552/v2

License: (c) (i) This work is licensed under a Creative Commons Attribution 4.0 International License. Read Full License 


\section{Abstract}

The authors have withdrawn this preprint due to erroneous posting. 